\title{
Long-term Intensive Locomotion Training with Wearable Hip-assist Robot in Stroke Patients: A Preliminary Study
}

Hwang-Jae Lee ${ }^{1,2}$, Su-Hyun Lee ${ }^{1}$, Dong-Seok Kim², Won Hyuk Chang', Bokman Lim³ , Byung-Ok Choi', Gyu-Ha Ryu', and Yun-Hee Kim ${ }^{1,2}$

Department of Physical and Rehabilitation Medicine, Center for Prevention and Rehabilitation, Heart Vascular Stroke Institute, Samsung Medical Center, Sungkyunkwan University School of Medicine, Seoul, Korea ${ }^{1}$, Department of Health Sciences and Technology, Samsung Advanced Institute for Health Science and Technology (SAIHST), Sungkyunkwan

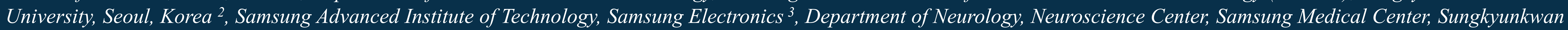

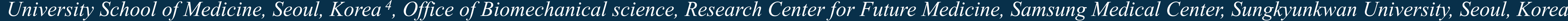

\section{Introduction}

- In recent years, several wearable robotic devices have been developed and used for rehabilitation training of individuals with hemiparesis after stroke. Most of these devices are computer controlled to improve lower limb movement, specially locomotion function.

- The purpose of this study was to investigate the long-term training effect of newly developed wearable hip-assist robot on locomotion function in stroke patients.

\section{The Wearable Hip-Assist Robot}

- The Gait Enhancing Mechatronic System (GEMS, Samsung Electronics Co. Ltd., Korea), which functions as a wearable hip-assist robot was used.

- The robot is developed to enhance walking performance and to increase the community mobility and social interaction in patients with gait disorders and elderly people.

- The robot consists of a pair of actuators that generate assistance power for the affected and unaffected hip joints, a hip brace around the waist, a pair of thigh frames that transmit assistance torque from the actuators to the thighs, and fabric belts at the ends of the thigh frame (Figure 1).
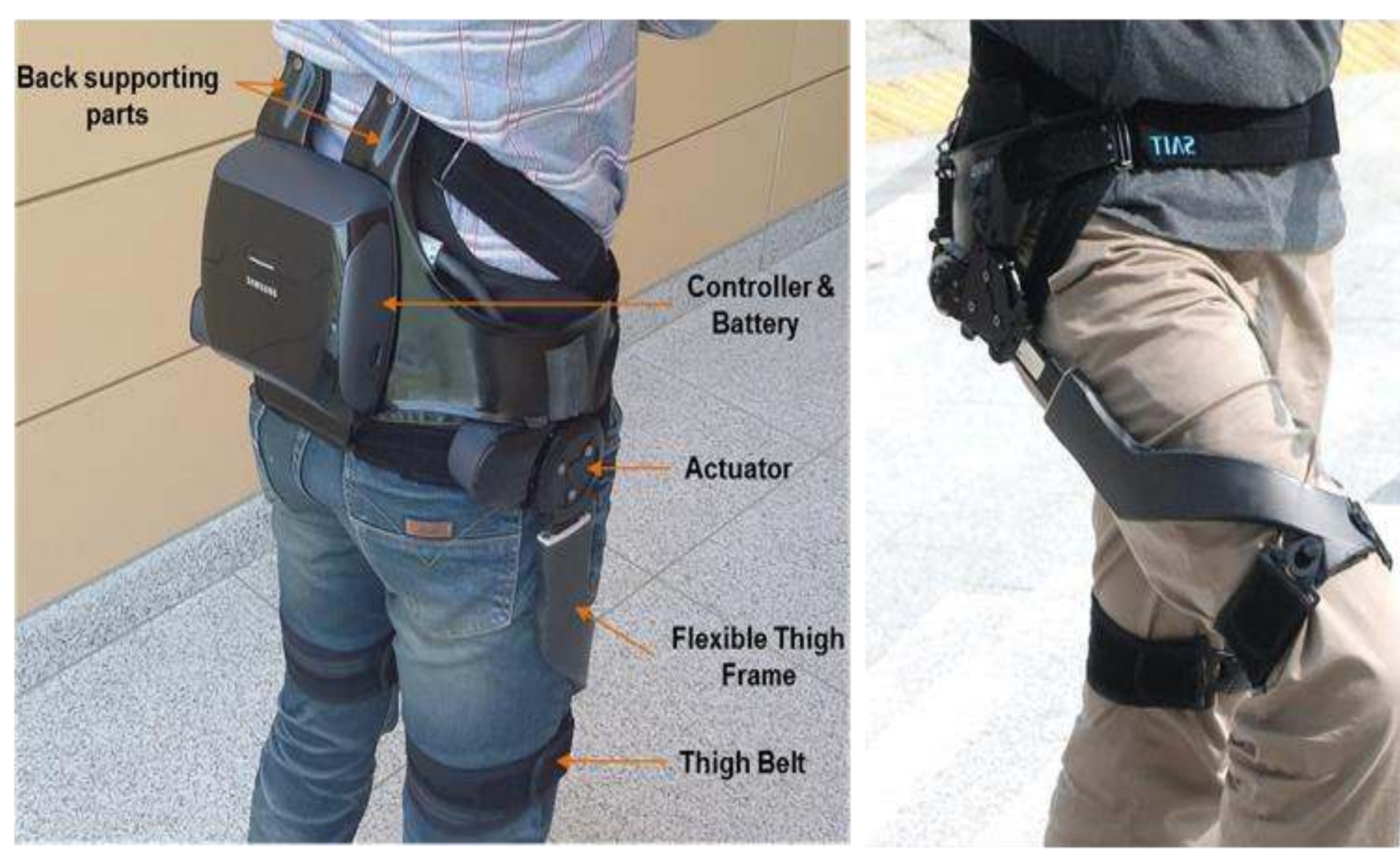

Gait Enhancing Mechatronic System (GEMS, Samsung Electronics Co., Ltd., Korea)

Figure 1. Wearable Hip-Assist Device, GEMS

- The assistance control method [DOFC (Delayed Output Feedback Control)] does not include a gait phase estimator as well as a reference data to generate torque profiles. However, it operates very robustly with respect to the change in the gait pattern by achieving self-adaptive control. The assistance torque is immediately applied following the movement of the user by reflecting the change in leg motion at each control cycle (Figure 2).
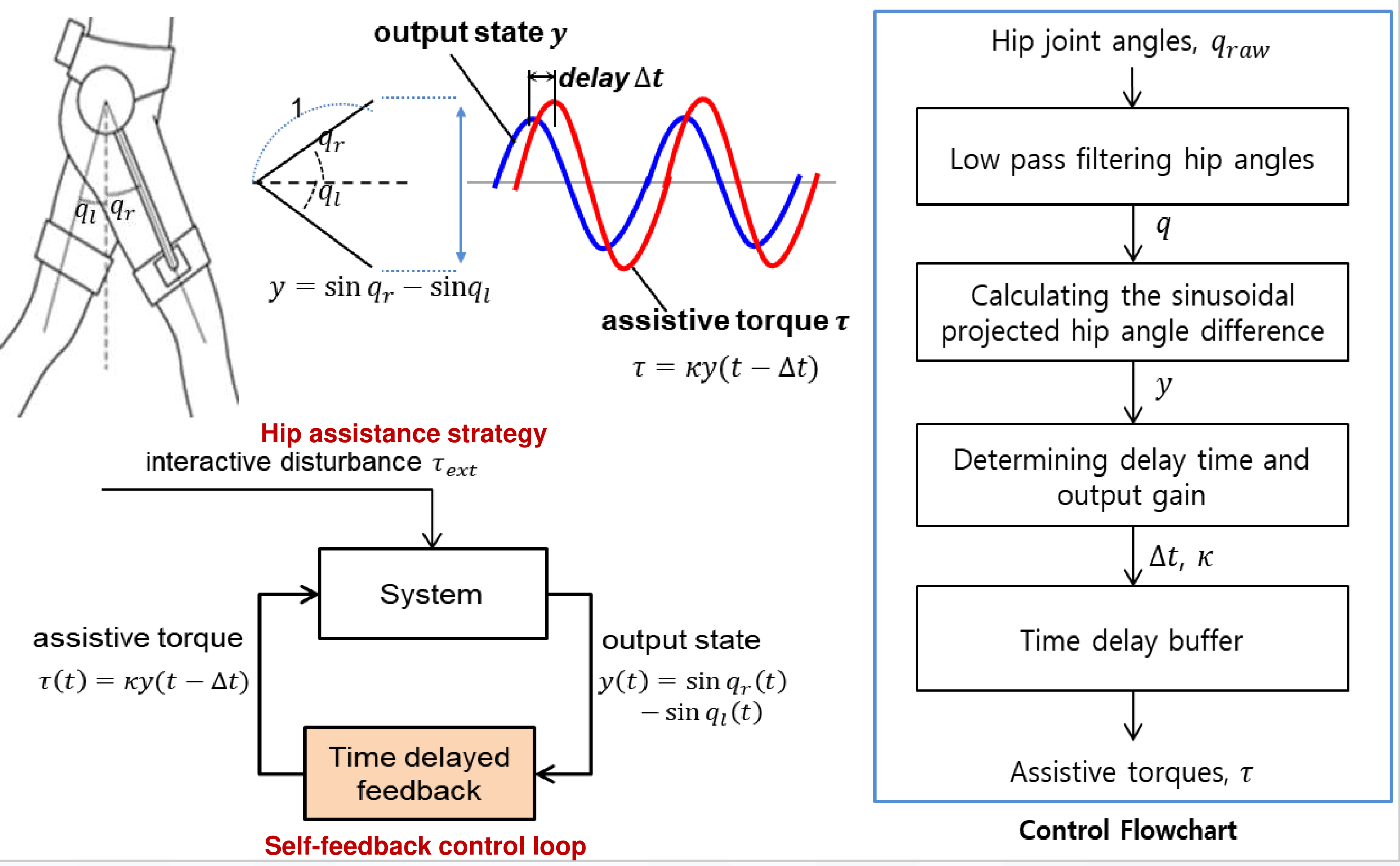

Figure 2. Gait assistance strategy with DOFC. $q_{r}$ and $q_{r}$ denote the right and left hip angles, respectively. $\kappa$ denotes the feedback gain.

\section{Participants and Experimental Protocol}

\section{Participants}

- Seven hemiparetic stroke patients (age means: $43 \pm 17.49,5$ males) participated and completed in this study.

- All participants received 45-minute gait training with GEMS in various overground environments for 24sessions during the consecutive 8 weeks. After 4 weeks, all participants underwent follow-up (Figure 3, 4).

\section{Measurements}

- Spatio-temporal parameters: 3D motion capture system with six infrared cameras (Motion Analysis Corporation, Santa Rosa, CA, USA)

- Muscle efforts: $12 \mathrm{ch}$ surface electromyography (sEMG) (Noraxon Inc., Scottsdale, AZ, USA)

- Cardiopulmonary metabolic efficiency: portable cardiopulmonary metabolic system (Cosmed K4B2, Rome, Italy)

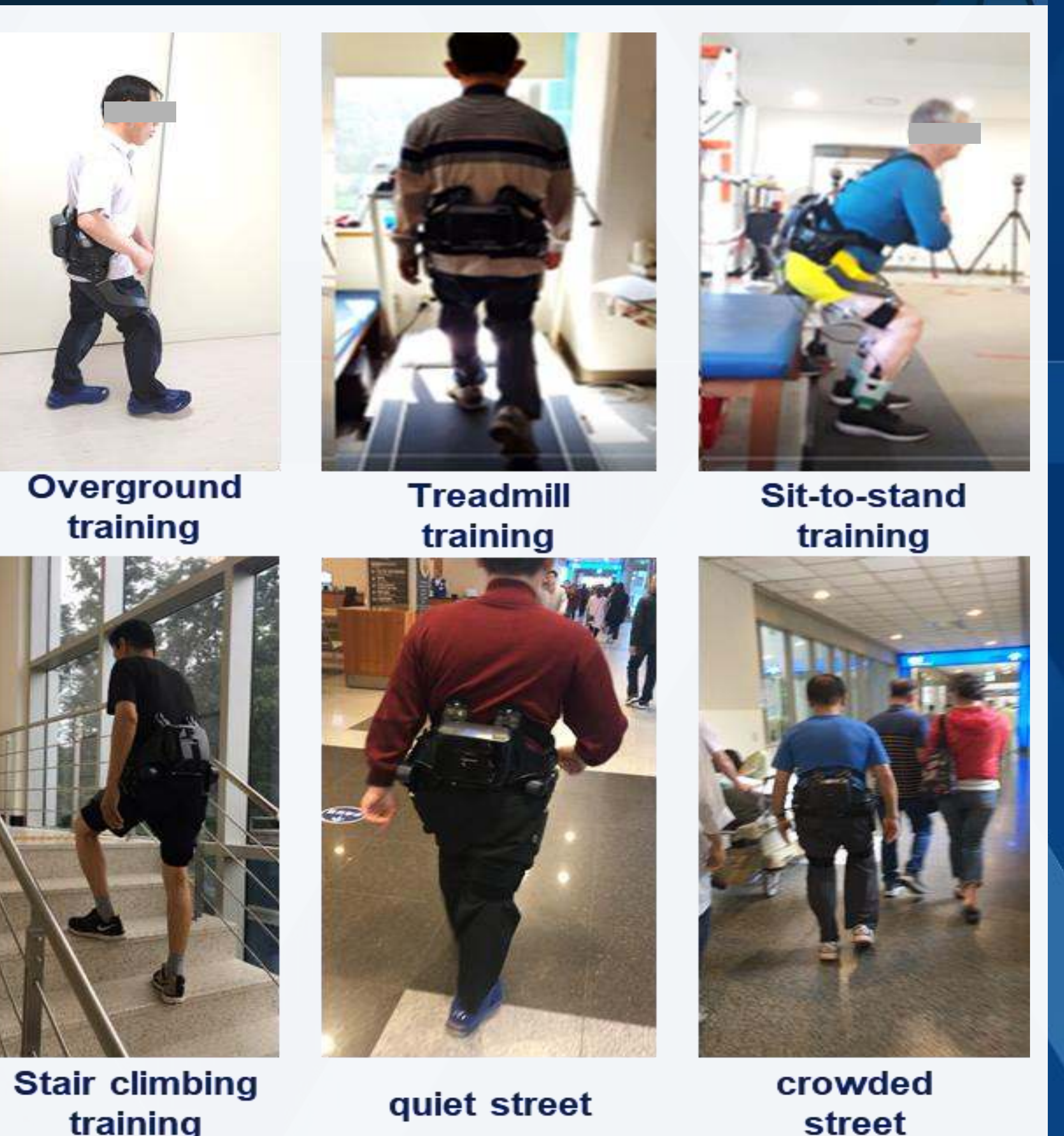

Figure 3. Locomotion training with GEMS in various overground environments

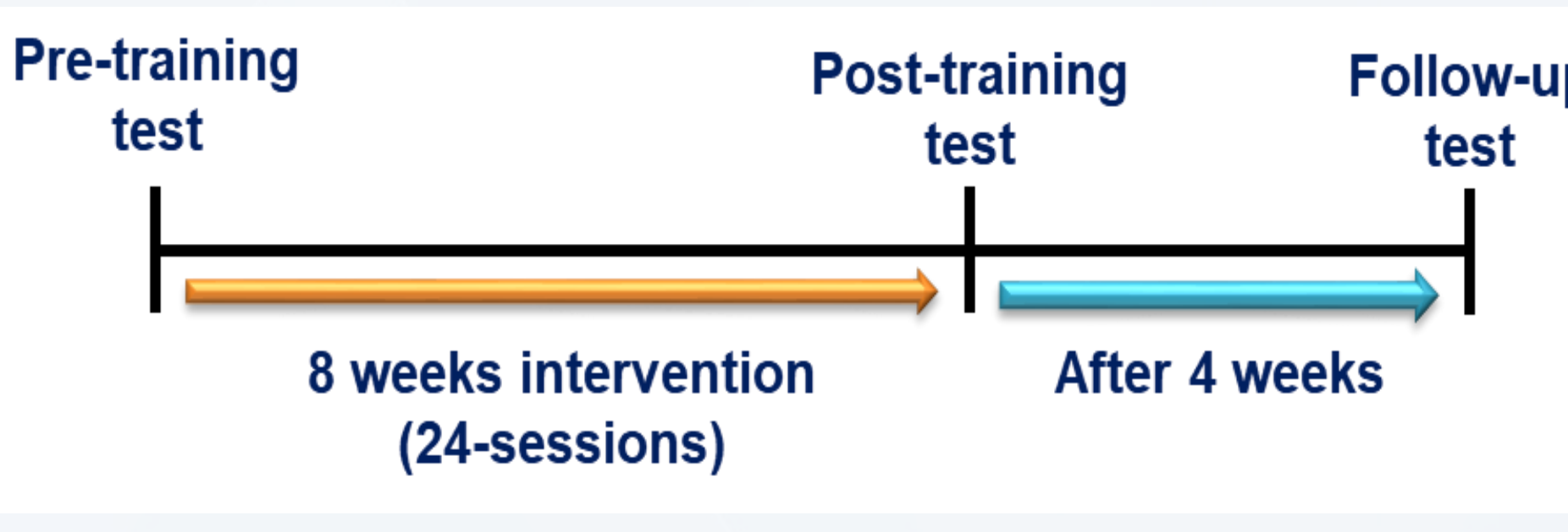

Figure 4. intervention protocol

\section{Results \& Conclusion}

- The 24-sessions of long-term intensive locomotion training with GEMS significantly improved gait function and gait symmetry of stroke patients $(P<$ 0.05).

- metabolic energy consumption during treadmill walking was significantly reduced after training (decreased 23.64\%). These gains were more reduced for 4 weeks after the cessation of training sessions (decreased $35.24 \%, \mathrm{P}<$

1. Muscle efforts (\%MVC) -Pre-training —Post-training -Follow-up

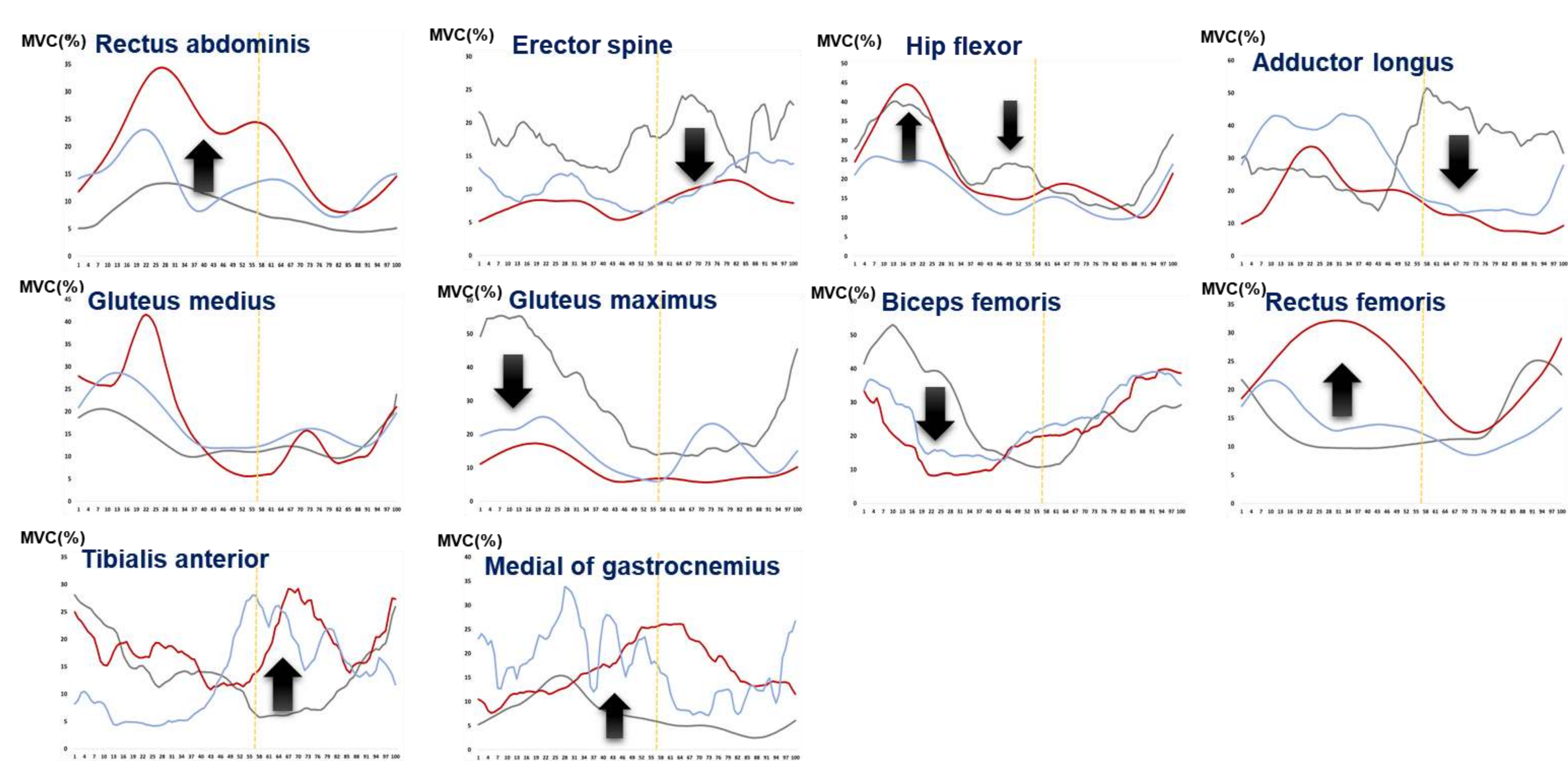

2. Spatio-temporal parameters
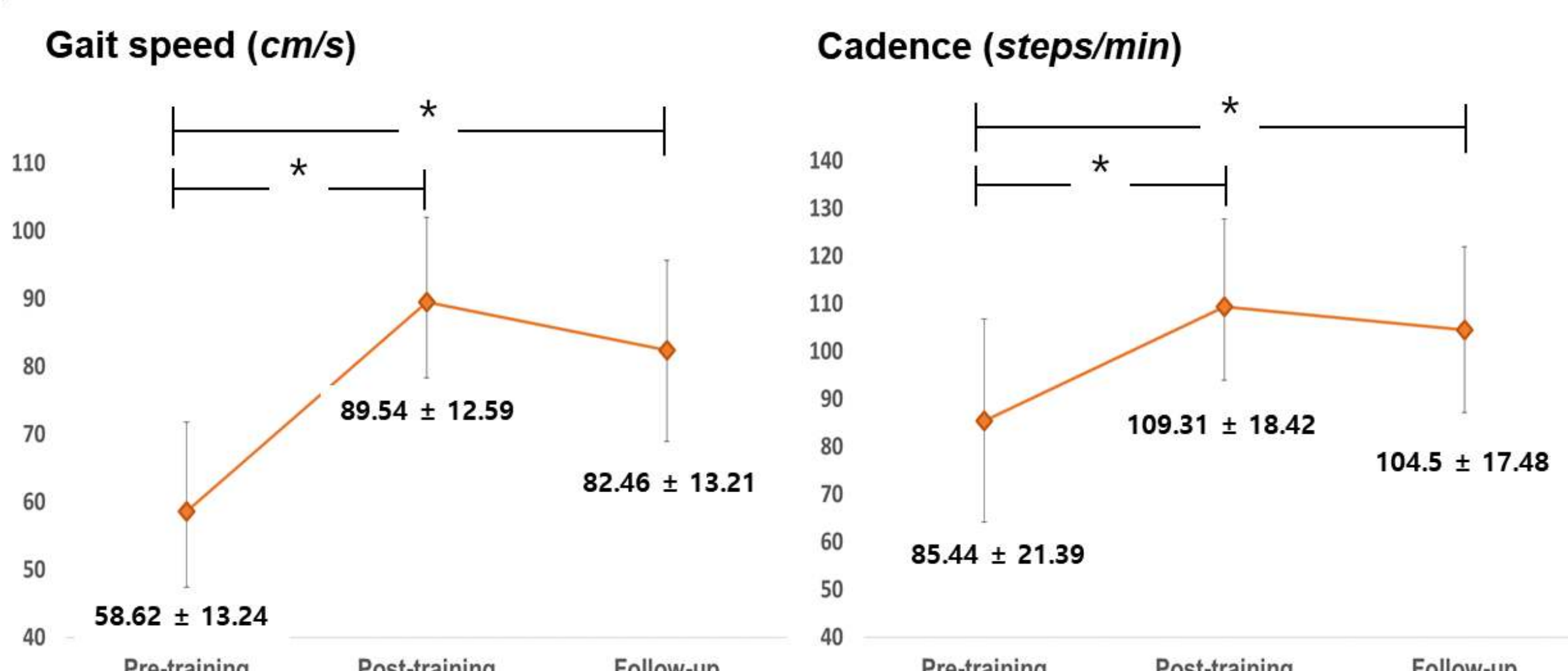

Stride length $(\mathrm{cm})$

The results of this preliminary study suggest that long-term intensive locomotion rehabilitation with the GEMS were tolerable and effective for improving gait function, gait symmetry, and cardiopulmonary metabolic efficiency during walking in the stroke survivors. Randomized controlled trial with larger participants is invited in near future. 